Revue Gouvernance

Governance Review

\title{
Gouvernance et incertitude de la téléphonie mobile
}

\author{
Le cas de la Belgique
}

Governance and Uncertainty of Mobile Telephony

The Case of Belgium

\author{
Caroline Deblander, Nathalie Schiffino et Louis Eeckhoudt
}

Volume 9, numéro 1, 2012

URI : https://id.erudit.org/iderudit/1038892ar

DOI : https://doi.org/10.7202/1038892ar

Aller au sommaire du numéro

\section{Éditeur(s)}

Centre d'études en gouvernance de l’Université d'Ottawa

ISSN

1912-0362 (numérique)

Découvrir la revue

Citer cet article

Deblander, C., Schiffino, N. \& Eeckhoudt, L. (2012). Gouvernance et incertitude de la téléphonie mobile : le cas de la Belgique. Revue Gouvernance / Governance Review, 9(1). https://doi.org/10.7202/1038892ar

\section{Résumé de l'article}

L'article analyse les choix de gouvernance opérés en Belgique quant au développement de la téléphonie mobile. Cette technologie connaît un succès économique et social avéré. Les effets à long terme, sur la santé et l'environnement, de la propagation des ondes électromagnétiques restent pourtant largement inconnus. Face à cette incertitude, les autorités publiques régulent le secteur en faisant intervenir une pluralité d'acteurs en réseaux (experts scientifiques, opérateurs, associations et citoyens). En théorie, elles peuvent appliquer trois principes : la prudence, la prévention et la précaution. En pratique, cette dernière s'impose comme une logique dominante. Reposant sur une approche interdisciplinaire, l'article adopte un angle d'analyse très spécifique. En effet, il mobilise des définitions économiques (univers risqué et " unique prior » versus univers incertain et « multiple prior »), pour montrer une partie de la complexité de la régulation politique. La difficulté de celle-ci réside notamment dans l'ambivalence de notre société prompte à s'alarmer des risques subis et collectifs (en l'occurrence, les antennes-relais) et tolérante aux risques individuels et choisis (dans le cas présent, les téléphones portables). Cette contribution est donc originale à deux égards. Premièrement, elle croise des connaissances des sciences économiques et des sciences politiques sur le risque pour décrire et expliquer la gouvernance de la mobilophonie. La seconde originalité réside dans le cas lui-même. En effet, la Belgique a adopté des normes plus restrictives que celles qui sont préconisées par les organisations internationales. Qui plus est, elle ne l'a pas fait principalement à l'échelon national, mais bien au niveau des Régions dans le cadre de nouveaux transferts de compétences entre l'État fédéral et les entités décentralisées, ces dernières interprétant différemment les principes de prudence, prévention et précaution. 


\title{
GOUVERNANCE ET INCERTITUDE DE LA TÉLÉPHONIE MOBILE
}

\author{
LE CAS DE LA BELGIQUE
}

par C. Deblander, N. Schiffino, L. Eeckhoudt

\begin{abstract}
INTRODUCTION
Comme Beck (1986) - le théoricien de la société du risque - le suggère, la régulation des risques modernes peut être appréhendée comme une forme d'irresponsabilité organisée. Alors que l'on assiste à une institutionnalisation du risque comme enjeu public, la multiplication des instances de régulation ne suffit pas à éviter des crises alors même que l'attribution des responsabilités se complexifie. Si la notion de risque est d'ailleurs devenue une référence pour qualifier une série de problèmes publics (Borraz 2008: 12), elle revêt pourtant des significations différentes selon les auteurs et les secteurs de politiques publiques (Jacob et Schiffino 2007). La formule provocante de Beck amène donc à réfléchir aux fondements individuels et collectifs de la décision publique.

Pour ce faire, notre contribution mobilise les notions de prudence, prévention et précaution dans le secteur de la téléphonie mobile. Analysées par les sciences économiques, ces 3P permettent de comprendre une partie des décisions politiques prises dans ledit secteur. Fondamentalement, l'équation intellectuelle (Paillé et Muchielli 2010 : 70) d'une recherche interdisciplinaire, alliant sciences économiques et politiques, peut amener une plus-value à une analyse de la gouvernance. Les sciences économiques apportent un éclairage positiviste de la régulation publique (Fischhoff et al. 1984). Les sciences politiques tendent à montrer la complexité de la fabrique des risques (Gilbert 2003), mettant en exergue la construction sociale du risque. En effet, le politique induit par définition conflit et coopération (Heywood 2007 : 4-12) avec tout ce que cela implique de subjectivité dans les liens complexes entre le social, la science et le politique pour gérer le risque.
\end{abstract}

Sur cette base, la question qui sert de fil conducteur à notre réflexion est la suivante : en quoi les apports respectifs des sciences économiques et des sciences politiques sur le risque nous permettent-ils de comprendre la régulation publique de la téléphonie mobile en Belgique, notamment au regard des $3 \mathrm{P}$ ?

Pour répondre à cette interrogation, l'article définit d'abord brièvement le risque et montre en quoi la mobilophonie constitue un enjeu de gouvernance. Ceci permet, dans un deuxième temps, d'articuler les notions économiques d'univers risqué et incertain respectivement aux effets thermiques et athermiques de la technologie. Il est alors possible d'articuler les 3P aux choix posés par les autorités politiques belges dans le secteur. L'article explique ainsi plus particulièrement les choix publics posés dans le cadre d'un Etat fédéral. Après avoir abordé la question des assurances et de la mutualisation des coûts, l'article conclut que, si le principe de précaution prévaut, c'est principalement en vertu d'une logique de gouvernance intégrant plusieurs profils d'acteurs au processus décisionnel, en vertu de l'incertitude qui caractérise la technologie.

Sur le plan méthodologique, l'article repose sur une analyse documentaire de la politique sur le long terme (de 1979 à 2011) et sur des entretiens. Les sources documentaires sont principalement constituées de textes de lois, d'archives, d'articles de presse et d'analyses 
d'auteurs dont certains sont mentionnés en bibliographie. Dix-huit entretiens semi-dirigés ont été menés auprès de mandataires politiques ayant participé à un processus de décision publique en la matière, de fonctionnaires en charge du suivi des dossiers, d'opérateurs privés constituant les leaders du développement économique du secteur, des principaux groupes d'intérêts mobilisés sur la problématique. Ces entretiens, d'une moyenne de 2 heures, ont été enregistrés et retranscrits in extenso. Ils ont fait l'objet d'une analyse lexicométrique à l'aide d'un logiciel d'analyse de données qualitatives. La récurrence des mots employés par les interviewés mais aussi les associations d'idées effectuées par eux ont confirmé les informations récoltées par l'analyse documentaire.

\section{DES LIENS COMPLEXES ENTRE POLITIQUE, SCIENCE ET SOCIÉTÉ}

Le risque est généralement défini comme la probabilité d'un évènement futur, positif ou négatif (Short 1984: 711). Lorsque les conséquences d'un évènement sont négatives, le risque peut être défini comme la probabilité qu'un danger survienne (Debia et Zayed 2003: 2). L'État moderne, parfois perçu comme omniscient et assurant la sécurité de sa population (Borraz $2008: 11$ ), définit de nouveaux champs d'activités et des dangers qui peuvent être assurés (Peretti-Watel 2001 :12-13). En référence à l'expansion de l'assurance publique et privée, on parle même d'assurantialisation de notre société (Ewald 1986). Nourrissant l'utopie de la sécurité et de la santé parfaite (Peretti-Watel 2009 :11), d'aucuns évoquent une "société du risque zéro ». Cette société implique une conception positiviste de l'homme et du savoir basée sur des certitudes scientifiques. Pourtant, dans des situations de risque potentiel, l'habituelle gestion des risques, reposant sur la théorie des choix rationnels, n'est d'aucun secours (Van Griethuysen 2004 : 56).

Le cas des ondes électromagnétiques (OEM) illustre bien la complexité due à la cohabitation de certitudes (connaissances amenées par le développement des sciences et techniques) et d'incertitudes telles que définies en statistiques (par exemple sur les effets à long terme et athermiques de la technologie). Le constat revêt une dimension particulière lorsque les technologies porteuses de risque se voient par ailleurs reconnaître une utilité sociale. Ainsi, de nombreuses qualités sont associées à la mobilophonie. D'abord, c'est un secteur économique rentable qui ne subit que marginalement les effets de la crise économique et financière actuelle. Ensuite, le téléphone portable est un objet consensuel pourvoyeur d'autonomie, permettant de s'affranchir des contingences spatiales liées à la téléphonie fixe. Enfin, il est synonyme d'indépendance (nous sommes libres de communiquer comme nous le souhaitons) et de sécurité (nous pouvons communiquer partout). Il est utile personnellement et professionnellement. L'accroissement exponentiel d'utilisateurs démontre, s'il en est besoin, le succès de cette technologie ${ }^{1}$.

En termes de santé publique, ses effets potentiels à long terme sont pourtant incertains. Et si le téléphone portable fait l'objet d'un large consensus concernant son utilité sociale, les antennes-relais sont moins acceptées socialement, même si elles sont considérées dans la réglementation comme des équipements de service public. Des riverains se plaignent d'être soumis de manière permanente à l' "electrosmog » produit par ces antennes. De plus, leur impact sur le paysage est présenté par des associations et des comités de quartier comme une

\footnotetext{
${ }^{1}$ En 2010, le nombre d'utilisateurs de téléphones portables dans le monde était de 5,3 milliards. The world in 2010 : facts and figures, ITU, http://www.itu.int/ITU-D/ict/material/FactsFigures2010.pdf [Accédé le le 5 juin 2011].
} 
défiguration du cadre de vie, ce qui peut engendrer une dévalorisation des habitations environnantes ${ }^{2}$.

C'est donc dans une tension permanente que les pouvoirs publics sont amenés à réguler. Ils prennent en considération l'expertise scientifique qui évalue au moins partiellement les risques à l'aide d'une série de facteurs quantifiables et souvent incomplets ainsi que la perception du risque par les citoyens. Or la littérature a démontré depuis longtemps (Starr 1969; Slovic 1987) que la perception du risque par les uns et les autres varie. Douglas et Wildavsky (1982) montrent que les individus perçoivent le risque comme étant d'autant plus négatifqu'il est involontaire, invisible et irréversible. Dans ce contexte, les pouvoirs publics sont amenés à faire des choix dits « de valeurs » en sciences économiques et à identifier le niveau de risque acceptable pour la population. Ils sont d'autant plus sourcilleux qu'une série de crises ont ébranlé l'image de l'État protecteur (Borraz 2008 : 32, 155) : les crises du sang contaminé, l'encéphalopathie spongiforme bovine (ESB), la dioxine, le scandale de l'amiante, par exemple. Ce sont autant d'épisodes qui ont ébranlé la confiance dans la capacité de l'État et de l'expertise à prévenir et gérer les risques (Godard $2000: 2$ ). Les pouvoirs publics tentent de rétablir de la confiance en appliquant les 3P, décodés par les sciences sociales, que sont la prudence, la précaution et la prévention.

Dans la foulée de ce qu'annonçaient Rosenau et Czempiel lorsqu'ils définissaient la gouvernance (1992: 4), ce contexte offre par excellence la possibilité d'une réelle gouvernance du risque, au-delà d'un gouvernement du risque qui formaterait la décision publique selon un processus traditionnel où domineraient les acteurs politico-administratifs. Ici, dans la logique définie par Jouve (2007 : 45), le décentrement d'une décision publique dominée par les pouvoirs publics se marque par l'émergence de nouveaux acteurs issus de la société civile et la mise en place de mécanismes de coordination ne passant plus uniquement par l'exercice de la domination mais au contraire par des mécanismes informels au sein de configurations institutionnelles plus souples. Tel que le montre Held (2007), cette gouvernance s'inscrit dans des jeux d'échelles. Par définition, cette notion implique que les changements contemporains de gouvernance sont intimement liés à une reconfiguration de l'État (Bherer et al. 2005). En Belgique, le processus de fédéralisation s'ajoute à celui de mondialisation pour expliquer que la régulation de la mobilophonie se réalise à plusieurs échelons : les organisations internationales, l'État fédéral et les Régions interagissent avec les experts scientifiques, les opérateurs privés et les citoyens mobilisés, comme le montre la suite de l'article.

\section{UNIVERS RISQUÉ : LES EFFETS THERMIQUES DE LA TÉLÉPHONIE MOBILE}

L'univers risqué est défini comme un univers dans lequel «l'agent sait quels évènements peuvent se réaliser dans le futur, et il connaît aussi la probabilité d'occurrence de chacun d'eux » (Peretti-Watel 2001: 20). En sciences économiques, il s'agit donc d'un cas d' 'unique prior $^{3}$ 'qui correspond, par analogie dans le cas des OEM, aux effets thermiques de la technologie. À certains niveaux d'exposition aux OEM, ceux-ci produisent un échauffement des tissus du corps humain, ce qui a été établi par des instances scientifiques comme

\footnotetext{
${ }^{2}$ Il existe plusieurs associations mobilisées sur les OEM : Teslabel, Beperk de straling, le Clag et le Grappe ; de plus, des comités de quartiers constitués de manière ponctuelle sont créés afin d'empêcher l'implantation d'antennes-relais dans leurs voisinages.

${ }^{3}$ Dans le cadre de la théorie des probabilités, il s'agit d'une situation où la distribution des probabilités sur les résultats est connue.
} 
l'International Commission on Non-Ionizing Radiation Protection (ICNIRP ${ }^{4}$ ), l'Organisation mondiale de la Santé (OMS) ou l'Union européenne se basant sur des éléments quantifiables pour adopter des normes de protection.

En 1998, l'ICNIRP a adopté une norme d'exposition aux OEM qui sert de référence partout dans le monde. Cette norme, non contraignante, est destinée à protéger les personnes contre les effets nocifs connus de ces champs (ICNIRP 1998). La limitation établie a été élaborée à partir d'une revue de la littérature scientifique publiée. Seuls les effets avérés ont été retenus comme fondements pour les valeurs limites d'exposition proposées. Selon cette commission, les seuls effets avérés sont des conséquences à court terme sur la santé, comme la stimulation des nerfs et des muscles périphériques, des décharges, des brûlures, ... causées par l'élévation de température des tissus ${ }^{5}$.

Selon le rapport établi par l'ICNIRP en 1998 (et confirmé en 2009), une exposition du corps humain à des valeurs de débit d'absorption spécifique (DAS) supérieures à $4 \mathrm{~W} / \mathrm{kg}$ engendre des effets sur la capacité de thermorégulation de l'organisme humain et provoque un échauffement des tissus. Sur base de cette valeur de DAS, une norme prévoyant une protection pour les travailleurs a été établie à $0,4 \mathrm{~W} / \mathrm{kg}$ de DAS moyen pour le corps entier. En ce qui concerne la population et partant du principe qu'elle est composée de personnes de tous âges, dont des groupes sensibles comme les enfants et les personnes âgées, la norme applicable au grand public prévoit un DAS de $0,08 \mathrm{~W} / \mathrm{kg}$. Que ce soit pour les travailleurs ou pour le public, un facteur de sécurité est appliqué afin de prendre en compte les incertitudes scientifiques sur les effets à long terme de la technologie.

Après que ces normes aient été établies en 1998 par l'ICNIRP, l’Union européenne (1999) est intervenue dans le secteur afin de garantir une protection sécuritaire et sanitaire uniforme en Europe ${ }^{6}$. Elle s'est exprimée par voie de recommandation ${ }^{7}$ (à destination du grand public) et par le biais d'une directive ${ }^{8}$ (à destination des travailleurs). Elle a par ailleurs souligné que les États membres peuvent déterminer des niveaux de protection supérieurs s'ils le souhaitent, ce qu'a fait la Belgique.

UNIVERS INCERTAIN : LES EFFETS ATHERMIQUES DE LA TÉLÉPHONIE MOBILE

L'univers incertain est défini comme un univers dans lequel « l'agent ne connaît ni les évènements possibles, ni a fortiori les probabilités d'occurrence » (Peretti-Watel 2001: 20). Pour prendre sa décision, l'agent doit lister les premiers et évaluer les secondes. Ce risque potentiel correspond, par analogie dans le cas de la mobilophonie, aux effets athermiques de la technologie ${ }^{9}$. Des chercheurs ont constaté des effets biologiques, mais " aucun mécanisme clair d'interaction onde-cellule n'a cependant été identifié pour des niveaux d'exposition non

\footnotetext{
${ }^{4}$ L'ICNIRP est une commission créée en 1992 compétente en matière de radiations non ionisantes. Cette commission est officiellement reconnue par l'OMS, l'Organisation Internationale du Travail (OIT) et par l'Union européenne.

5 ICNIRP (1999), Use of the ICNIRP EMF guidelines, http://www.icnirp.de/documents/Use.htm [Accédé le 22 septembre 2008].

${ }^{6}$ Rapport sur la proposition de recommandation du Conseil relative à la limitation de l'exposition du public aux champs électromagnétiques $(0 \mathrm{~Hz}-300 \mathrm{GHz}), 22$ février 1999, p.16.

${ }^{7}$ Recommandation du Conseil du 12 juillet 1999 relative à l'exposition relative à l'exposition du public aux champs électromagnétiques, Journal Officiel des Communautés européennes, L 199/55, 30 juillet 1999.

${ }^{8}$ Directive 2004/40/CE du Parlement européen et du Conseil du 29 avril 2004 concernant les prescriptions minimales de sécurité et de santé relatives à l'exposition des travailleurs aux risques dus aux agents physiques (champs électromagnétiques), Journal Officiel des Communautés européennes, L 184/1, 24 mai 2004.

9 «Les effets non thermiques ou 'athermiques' apparaitraient à des niveaux d'exposition non thermiques, pour lesquels le corps peut réguler sa température, sans que l'on observe macroscopiquement d'augmentation de celle-ci » (Afsset 2009a: 9).
} 
thermiques » (Afsset 2009 :11). En sciences économiques, il s'agit donc d'un cas de 'multiple prior $^{10}$ ' qui relève du domaine de l'incertitude, au sens que lui confèrent les probabilités. Dans le secteur de la téléphonie mobile, les évènements possibles identifiés sont notamment des effets génotoxiques, apparition de cancers, effets délétères sur le système nerveux, dysfonctionnement du système immunitaire, perturbation du système cardio-vasculaire ${ }^{11}$. Ces effets potentiels ont fait l'objet d'études mais, à l'heure actuelle, le faisceau de preuve est insuffisant.

A l'évidence, les pouvoirs publics ne mobilisent pas uniquement l'expertise scientifique. Les choix politiques prennent en compte le degré de risque qu'une société est disposée à accepter et à assumer (November et November 2004 : 176), les perceptions subjectives du risque et de l'incertitude (Peretti-Watel 2009 : 29). Dans un tel contexte, les pouvoirs publics peuvent opter pour trois stratégies différentes : la prudence, la prévention ou la précaution.

\section{LA PRUDENCE : UNE RÉGULATION PUBLIQUE ENTRE ANTICIPATION ET INCERTITUDE}

Adopter une attitude de prudence sociale consiste à agir précocement, en accordant plus de poids aux perspectives dites pessimistes qu'aux perspectives dites optimistes (Van Griethuysen 2004 : 63). Il est communément accepté que prévention et précaution sont filles de la prudence. Celle-ci s'impose dès que des décisions présentent des risques avérés ou potentiels (Hunyadi 2004 : 17).

Si l'on applique la notion de prudence à la régulation belge des OEM, une attitude prudente s'exerce à l'égard du terminal de téléphonie mobile et s'illustre au début de la régulation publique par la loi fédérale de $1998^{12}$. Celle-ci établit (a) la protection de l'environnement contre les effets ou risques d'effets nocifs de certains produits mis sur le marché, (b) la protection de la santé contre les effets ou risques d'effets nocifs de certains produits mis sur le marché.

La stratégie de prudence se vérifie également dans le fait que les téléphones portables subissent une série de tests avant leur commercialisation, afin de pouvoir être mis sur le marché. Ils doivent notamment respecter des normes de rayonnement maximal. Ces normes sont définies à l'échelon international et européen.

\section{LA PRÉVENTION : UNE RÉGULATION PUBLIQUE ENTRE RATIONALITÉ ET ÉVITEMENT}

La prévention est inhérente au risque au sens économique du terme. Dans une telle perspective, la prévention est définie comme une «conduite rationnelle face à un mal que la science peut objectiver et mesurer » (Godard 1997: 121). Cette notion est dépendante de celle de certitude scientifique et conduit à l'application de la théorie des probabilités dans les choix de société. Dans ce cas, les mesures préventives résultent d'une analyse comparative des avantages et inconvénients associés à différents scénarios.

Dans le cas des OEM, la prévention s'illustre par des campagnes visant à exclure l'usage du téléphone mobile par les enfants dont le cerveau est encore en développement. En 2009, la ministre régionale de la Santé, au sein du gouvernement wallon, a ainsi déclaré que

\footnotetext{
${ }^{10}$ Situations dans lesquelles les distributions de probabilités ne sont pas connues.

11 Selon les degrés de plausibilité pris en compte, différentes hypothèses sont avancées concernant les effets athermiques de la technologie. Nous nous sommes référés aux effets possibles identifiés par l'Afsset parce qu'elle constitue l'expertise généraliste la plus actuelle à ce jour.

${ }^{12}$ Loi du 21 décembre 1998 relative aux normes de produits ayant pour but la promotion de modes et de consommation durables et la protection de l'environnement et de la santé, Moniteur belge, 11 février 1999.
} 
« les études scientifiques montrent que plus on utilise le GSM lorsqu' on est jeune, plus il y a de risque élevé de dégâts sur la santé liés au rayonnement ${ }^{13}{ }^{2}$.

De plus, d'après l'Institut belge de sécurité routière (IBSR), l'utilisation d'un téléphone au volant augmente de 75 pour cent le risque d'accident ${ }^{14}$, c'est pourquoi son usage au volant est proscrit depuis $2000^{15}$. Des campagnes de prévention sont réalisées par l' IBSR afin de sensibiliser la population à ce risque ${ }^{16}$. Si cet exemple ne porte pas sur les OEM en tant que telles, il illustre par contre le type d'arguments qui peuvent étayer les décisions publiques.

Afin de gérer le risque, la prévention n'est pas le seul instrument possible, l'assurance peut aussi être mobilisée. La prévention vise à éviter le risque en réduisant autant que possible sa probabilité d'occurrence et la gravité des problèmes qu'ils peuvent induire tandis que l'assurance, antidote du risque, compense les dommages lorsqu'un risque se réalise (PerettiWatel 2009: 19). À l'heure actuelle, la téléphonie mobile ne fait pas l'objet de couverture assurantielle; nous y reviendrons. Remarquons d'ores et déjà que l'assurance peut anesthésier, voire même devenir le concurrent de la prévention. Si un individu est assuré contre un risque, il pourra se comporter différemment que s'il n'était pas couvert contre ce risque. L'assurance et la prévention peuvent être conjuguées afin de gérer le risque, mais dans les cas d'incertitude, cette conception va être supplantée par la précaution dont la caractéristique principale repose sur le caractère incertain.

\section{La PrÉCAUTION : UN PRINCIPE DOMINANT ET CONTROVERSÉ}

La précaution, définie comme l'anticipation d'un risque potentiellement grave (Dupont 2007 : 374-376), est apparue comme une norme de gestion en matière d'environnement vers 1980 et a été consacrée lors de la conférence de Rio en 1992 (Kourilsky 2002 : 9). La précaution prend en considération les coûts et les bénéfices de l'action et de l'inaction (EEA 2008 : 13). Elle est utilisée pour réduire les dangers potentiels, et ce, préalablement à la preuve du dommage causé. Sous l'impulsion de la crise de la vache folle, ce principe s'est progressivement étendu aux problématiques sanitaires (Ewald 2009 : 19). Cependant, cette extension nuit à la précision du concept (Hunyadi 2004 : 10) et conduit à des interprétations différentes au sein du monde scientifique et au sein de la société civile. Certains auteurs établissent une adéquation entre précaution et inversion de la charge de la preuve, c'est-à-dire que la précaution consiste en une présomption de nocivité et serait un principe d'inaction. D'autres auteurs désapprouvent cette définition et considèrent que ce principe demande une action anticipative, mais proportionnée, ce qui implique une prise en compte précoce des hypothèses de risque sans bloquer l'innovation et en renforçant le besoin de recherche.

Au sein de la société civile, la précaution est souvent mobilisée comme une solution, mais cette mention est la plupart du temps rhétorique sans indication explicite du contenu que l'on y associe. «Inter Environnement Wallonie », une plateforme d'associations environnementales, a pourtant précisé le contenu d'un tel scénario. Le groupe d'intérêt

\footnotetext{
${ }^{13}$ Belga (2009), « Tillieux dit stop au GSM à l'école primaire », Le Soir, 28 novembre.

${ }^{14}$ IBSR, Téléphoner au volant, http://www.bivv.be/main/OnzeCampagnes/Archief/DetailCampaign.shtml?detail=887357440\&language=fr [Accédé le 3 février 2010].

${ }^{15}$ Arrêté royal du 24 juin 2000 portant règlement général sur la police de la circulation routière, Moniteur Belge, 28 juin 2000.

${ }^{16}$ D. Fontaine. 2009. « GSM au volant, crash au tournant », Le Soir, 31 juillet.
} 
« demande une application sans concession du principe de précaution et l'adoption du niveau d'exposition le plus faible recommandé par les scientifiques, soit $0,6 \mathrm{~V} / \mathrm{m}^{17} \gg$.

De manière générale, la définition des normes pour les antennes-relais en Belgique découle de l'application du principe de précaution. Singulièrement, ce qui dénote l'originalité de la régulation publique belge, c'est que les normes définies aujourd'hui par les Régions sont plus restrictives $(3 \mathrm{~V} / \mathrm{m}$ pour une fréquence de référence de $900 \mathrm{MHz})$ que celles établies auparavant par l'État fédéral $(20.6 \mathrm{~V} / \mathrm{m})$ et par les organisations internationales $(41,2 \mathrm{~V} / \mathrm{m})$.

Cette régulation plus restrictive découle de la prise en considération des inquiétudes citoyennes, de l'avis d'un comité d'experts en matière sanitaire (le Conseil Supérieur de la Santé, CSS), et de la volonté des Régions de s'affirmer par rapport à l'État fédéral. En effet, les Régions invoquent leurs compétences en matière environnementale pour réduire les normes d'émission des antennes-relais, encouragées en cela par les associations environnementales et des comités de citoyens. Comme le précisaient les définitions de la gouvernance (Jouve 2007; Held 2007), des acteurs influencent donc nouvellement le processus décisionnel qui glisse de l'échelon fédéral à l'échelon régional.

\section{À LA RECHERCHE DE CERTITUDES EN VUE DE LA DÉCISION PUBLIQUE}

La décision en contexte d'incertitude prend en compte trois paramètres (Afsset 2009b) : la plausibilité, la réductibilité et l'observabilité. Lorsque les pouvoirs publics décident de réguler la mobilophonie, ils s'interrogent sur la possibilité que le risque sanitaire et environnemental se concrétise et engendre des effets négatifs. Ils questionnent la possibilité de les réduire, sur base de données observables étayant les décisions publiques.

Plusieurs degrés de plausibilité sont identifiés. La plausibilité peut être faible si les hypothèses de travail relatives au risque ne sont pas étayées par des données factuelles. Elle peut être moyenne si les hypothèses sont jugées recevables, mais nécessitent encore des recherches ciblées. Elle peut être forte si les hypothèses ne sont pas prouvées expérimentalement, mais que la majorité de la communauté scientifique partage la conviction du risque (Godard 2003 : 1253).

En fonction des divers degrés d'incertitudes, c'est-à-dire dans le cadre d'un principe de précaution, plusieurs attitudes concrètes sont envisageables : la vigilance, la recherche, l'information, les restrictions d'usage et l'interdiction temporaire ou permanente (Afsset 2009b). Nous allons illustrer ces différentes attitudes dans le cas de la mobilophonie en Belgique. Elles éclairent la façon dont le principe de précaution peut être opérationnalisé et montrent comment la régulation en Belgique a été adoptée à plusieurs échelons de pouvoir.

De nombreux programmes de recherche sur les OEM ont été lancés au niveau européen et international. A l'échelon international, l'OMS a lancé le projet international CEM afin d'encourager la recherche et promouvoir une communication coordonnée pour répondre aux inquiétudes de citoyens. L'étude Interphone a quant à elle réuni treize pays : France, Royaume-Uni, Allemagne, Italie, Danemark, Suède, Norvège, Finlande, Canada, Japon, Nouvelle-Zélande, Australie, Israël.

A l'échelon européen, l'étude Reflex a examiné les effets des OEM sur la santé. Le Scientific Committee on Emerging and Newly Identified Health Risks (SCENIHR) participe aussi à la mise à jour des recherches. Cet organe de la Commission européenne ne réalise pas de recherche, mais il est composé de spécialistes qui analysent et évaluent la littérature

\footnotetext{
17 P. Titeux. 2009, « ondes électromagnétiques : la loi au service de la santé », Inter-Environnement Wallonie, http://www.iewonline.be/spip.php?article2775 [Accédé le 11 février 2010].
} 
scientifique disponible sur le sujet et préconise au besoin une adaptation des normes en vigueur. De même, plus récemment, le Centre international de recherche sur le cancer (CIRC) a évalué la littérature scientifique sur l'usage du téléphone portable, ce qui a conduit à un classement des téléphones portables comme étant «peut-être cancérogènes ${ }^{18}$ ».

De plus, au niveau belge, tant fédéral que régional, plusieurs groupes de recherche existent dont le Belgian BioElectroMagneticGroup (BBEMG) et le Vlaams Instelling voor Technologisch Onderzoek (VITO). En outre, de nombreuses recherches sont menées dans les universités du pays ${ }^{19}$.

Des informations sont accessibles aux citoyens, dont un site Internet ${ }^{20}$, qui présente la réglementation en vigueur, expose des détails techniques sur la technologie et prodigue certains conseils aux utilisateurs comme l'utilisation d'un kit oreillette. De plus, la ministre fédérale de la Santé publique a édité en 2008 un guide édictant des recommandations et des conseils pratiques pour un usage raisonnable du portable ${ }^{21}$.

En ce qui concerne l'utilisation du portable par les jeunes, le Centre de Recherche et d'Information des Organisation de Consommateurs (CRIOC) mentionne que : "L'utilisation du téléphone mobile pourrait, entre autres chez les enfants, causer des problèmes de santé (risques de cancers, surdité après mauvaise utilisation, etc.). Aujourd'hui, il n'y a pas (encore) de vraies preuves que la radiation électromagnétique soit nuisible, mais il n'est pas non plus prouvé que tel n'est pas le cas. Il serait avisé surtout pour les enfants, d'appliquer le principe de précaution : une utilisation prudente du GSM réduit la charge d'irradiation à laquelle ils seront exposés ${ }^{22} »$.

De plus, un cadastre recensant les antennes-relais sur le territoire belge est disponible. Diverses agences administratives, tant à l'échelon fédéral que régional, tiennent ce cadastre à jour et réalisent des contrôles sur le terrain afin de vérifier que les normes de rayonnement sont respectées. En 2009, suite à une action introduite par la Région bruxelloise avec le soutien d'associations environnementales et de comités de citoyens, la Cour Constitutionnelle a rendu un arrêt qui a marqué la régionalisation de la compétence en matière d'OEM. Auparavant, la compétence était détenue par l'autorité fédérale et les contrôles étaient effectués uniquement par l'Institut belge des services postaux et des télécommunications (IBPT). Désormais, trois agences administratives sont chargées de réaliser les contrôles. Ce sont l'IBPT en Région flamande, l'Institut bruxellois pour la gestion de l'environnement (IBGE) en Région bruxelloise et l'Institut scientifique de service public (ISSeP) en Région wallonne.

En outre, au niveau local, pour l'implantation de nouvelles antennes-relais, une enquête publique est organisée à destination des riverains dans le cadre des procédures de permis d'urbanisme. Cette procédure d'information et de consultation permet aux citoyens de marquer leur désaccord, le cas échéant, sur l'implantation d'antennes-relais qu'ils n'ont pas choisies (risque subi). Néanmoins, cet avis n'est pas contraignant pour les autorités publiques.

Cependant, nonobstant ces exemples d'information à destination du public, le secteur des télécommunications estime qu'il existe un déficit d'informations au regard de ce qui peut être pratiqué dans d'autres pays, ce qui est attesté par un Eurobaromètre consacré aux OEM. 69\%

\footnotetext{
${ }^{18}$ s.a. (2011) « Pour l'OMS, le téléphone portable est peut-être 'cancérogène' », Le Monde, $1^{\text {er }}$ juin.

${ }^{19}$ On peut notamment mentionner la thèse de Dirk Adang sur l'impact des OEM sur les rats (UCL).

${ }^{20}$ www.infogsm.be

${ }^{21}$ Sénat de Belgique, Question n ${ }^{4}$-352 adressée à la ministre des Affaires sociales et de la Santé publique, 15 février 2008.

${ }^{22}$ CRIOC, Jeunes et GSM, http://www.oivo-crioc.org/files/fr/4662fr.pdf [Accédé le 15 janvier 2010].
}

Revue gouvernance automne 2012 
des Belges se disent insatisfaits du manque d'information et du manque d'objectivité de celleci (Commission européenne 2007 : 17).

Des restrictions d'usage sont également d'application. Arguant du principe de précaution, le ministre fédéral de la Protection des consommateurs s'est ainsi opposé à la mise sur le marché d'un téléphone portable conçu pour les enfants ${ }^{23}$. De plus, des restrictions d'usage existent en ce qui concerne la définition des normes fédérales applicables aux téléphones portables, fixant l'indice maximal de DAS à $2 \mathrm{~W} / \mathrm{kg}$.

L'interdiction temporaire concerne l'utilisation des portables dans des situations spécifiques. Le portable est proscrit dans certains services hospitaliers, notamment dans les unités de soins intensifs et de réanimation. Cette interdiction est justifiée par le risque d'interférences pouvant entraver le bon fonctionnement des appareils médicaux. L'usage du portable est proscrit dans les avions pour la même raison que dans les hôpitaux. Enfin, une interdiction plus controversée relève de l'utilisation du portable dans les stations-service. Cette interdiction date de l'apparition des portables analogiques ( $1^{\text {ère }}$ génération) et est justifiée par le risque d'explosion des vapeurs d'essence, mais ne repose sur aucune étude scientifique valide (Burgess 2006: 159). Ces exemples nous éloignent certes de la stricte application des OEM dans le seul secteur de la santé publique, mais ils attestent de la nécessité de réguler la mobilophonie dans une logique de gouvernance puisque ses enjeux sont multiples.

\section{COMMENT QUALIFIER LA RÉgulation PUbliQue de la tÉlÉPHONIE MOBILE EN BELGIQUE?}

Dans une dynamique de gouvernance associant plusieurs acteurs au processus de décision publique, la régulation de la téléphonie mobile par les Régions répond à une logique de précaution. Il est possible d'expliquer une telle régulation par au moins trois facteurs. Cidessus, nous avons déjà développé le contexte historique des crises sanitaires et les inquiétudes de la population. À ces deux facteurs s'ajoutent les «best practices » observables dans d'autres pays. En effet, sans aller jusqu'à un «policy transfer» (Dolowitz et Marsh 2002), les associations ou les mandataires politiques que nous avons interviewés, particulièrement à l'échelon régional qui régule de façon plus stricte le secteur, mentionnent plusieurs exemples à suivre :l'Autriche, la Grèce, le Liechtenstein, le Luxembourg, la Pologne et la Suisse appliquent ainsi des régulations dont la Belgique s'inspire. La norme luxembourgeoise semble notamment avoir influencé la définition d'une norme comparable en Région wallonne.

Malgré le caractère confus du principe de précaution (Kourilsky $2002: 15$ ), il permet aux Régions de concilier le développement technologique et économique avec la protection de la santé publique. Cette idée de compromis n'est pas nouvelle et s'inscrivait déjà dans la norme fédérale. Néanmoins, à cet échelon de pouvoir, le compromis nécessitait la prise en compte à la fois des intérêts francophones, plus enclins à adopter des normes restrictives, et des intérêts néerlandophones, privilégiant le développement économique du secteur. Ce compromis a été longuement contesté, faisant l'objet de recours en justice de la part des associations et conduisant les parlementaires régionaux à se saisir du problème. Au niveau régional, les décideurs politiques sont en effet proches des associations environnementales et des comités de citoyens. En captant la compétence, les Régions ont pu s'affirmer dans le cadre du processus de fédéralisation et réguler la mobilophonie en faisant écho aux inquiétudes du tissu associatif mobilisé sur la problématique.

\footnotetext{
${ }^{23}$ Belga (2008), « Magnette dit non aux GSM pour enfants », Le Soir, 4 novembre.
} 
Plus globalement, les différents compromis auxquels parviennent les décideurs politiques dépendent du niveau de risque acceptable au sein de la société, des rapports de pouvoir et des intérêts des acteurs. Les opérateurs (favorables à la technologie et mobilisés à l'échelon fédéral) versus les associations environnementales et les citoyens électro-sensibles (mobilisés principalement aux échelons régional et local), par exemple, ne perçoivent pas le risque de la même manière et jugent différemment leur niveau d'acceptabilité (Hergon et al. $2004: 135)$.

L'acceptabilité augmente lorsque le risque est assumé volontairement, associé à des bénéfices perceptibles immédiatement, et connu (Hergon et al. 2004 : 136). C'est le cas de la majorité de la population belge appréciant les téléphones portables. Plus spécifiquement, en témoigne par exemple le nombre important d'utilisateurs du téléphone portable au volant alors qu'un risque accru d'accident est avéré ${ }^{24}$. En effet, les sources d'exposition volontaires sont plus acceptées socialement que les prises de risque involontaires (Starr 1969; Slovic 1987). Si une exposition est perçue comme imposée et involontaire la perception du risque augmente (Kheifets et al. 2006 : 50). Ainsi, les antennes-relais sont moins acceptées en raison de leur caractère subi, mais peut-être aussi, alors que les ondes sont invisibles et immatérielles, parce que les antennes-relais sont visibles. Ce sont autant d'éléments que les pouvoirs publics prennent en considération dans la régulation publique du risque. Comme le montre la section sur les assurances ci-dessous, celle-ci fait également intervenir des conceptions liées au secteur privé.

\section{LES ASSURANCES : NOCIVITÉ OU RENTABILITÉ?}

Le fait que les assureurs privés ne veulent pas compenser les dommages potentiels liés aux OEM peut déclencher des interrogations au sein de la population. Il ressort des entretiens que nous avons réalisés que les associations voient dans l'absence de couverture de ces risques par les compagnies d'assurance une preuve de la nocivité de la technologie.

Cependant, l'absence d'assurance privée peut résulter d'autres causes. Idéalement, l'assurance privée couvre des risques de perte (à l'opposé des risques spéculatifs) dont on peut assez bien quantifier les deux versants : d'une part, on prévoit le nombre d'accidents qui auront lieu chaque année, d'autre part, on évalue le coût financier des dommages correspondants (Peretti-Watel 2001: 9). De plus, l'assurance privée préfère couvrir des risques indépendants et se méfie des risques positivement corrélés où le dommage, s'il survient, touche une majorité d'assurés.

Étant donné que l'assurance des OEM ne possède pratiquement aucune des caractéristiques intéressantes pour l'existence d'un marché privé de l'assurance, les assureurs s'en tiennent à l'écart. Cette absence de couverture ne signifie rien en termes de (non) nocivité des OEM. En effet, dans le cas d'un évènement dont les conséquences peuvent être soit négatives, soit positives, les assurances privées ont moins tendance à intervenir, parce qu'il existe une possibilité de spéculation de la part de l'assuré et parce que le risque potentiel étant collectif et généralisé, elles ne peuvent faire jouer le principe de diversification. Si l'évènement positif ou négatif doit malgré tout être pris en charge, cela se fera via l'assurance collective (sécurité sociale). C'est alors ici qu'interviennent directement les pouvoirs publics en vertu, nous l'avons expliqué, d'un principe de précaution.

En Belgique, la sécurité sociale couvre la population contre les maladies répertoriées par les pouvoirs publics dans l'assurance nationale des maladies et invalidités. Des traitements médicaux et pharmaceutiques contre certains symptômes associés aux OEM, comme les

\footnotetext{
24 s.a. (2011), « GSM au volant : nombre record d'amendes », Le Soir, 9 mars.
} 
céphalées, peuvent faire l'objet d'un remboursement par la sécurité sociale. Néanmoins, l'électro-sensibilité n'étant pas reconnue comme un handicap, les divers dispositifs destinés à atténuer les OEM (peintures, films métalliques, par exemple) ne sont pas remboursés, alors

que c'est le cas en Suède. À l'heure actuelle, l'assurance nationale des maladies et invalidités n'établit par ailleurs pas de lien entre le cancer du cerveau et l'utilisation de la téléphonie mobile. Il sera très intéressant d'étudier l'évolution de la régulation publique pour déterminer dans quelle mesure, notamment en lien avec l'attitude des assureurs privés, elle évoluera d'un principe de précaution à celui d'une prévention.

\section{CONCLUSION}

Grâce à l'étude d'une politique du risque particulière, nous illustrons les nombreux écueils auxquels les décideurs publics sont aujourd'hui confrontés. Dans le cas des OEM, le recours à l'expertise apparaît comme un outil de légitimation partielle de la décision publique. La complexité des rapports entre politique, science et société apparaît dans les divisions qui se dessinent entre les experts scientifiques globalement persuadés de l'innocuité des OEM et ceux qui dénoncent la nocivité des OEM. Ces experts, prenant différemment en compte le risque et l'incertitude, se différencient entre eux sur le degré de plausibilité des hypothèses prises en compte. La société civile est elle-même traversée par des oppositions entre une majorité soutenant la technologie et une minorité questionnant ses potentiels effets. Face aux perceptions divergentes de ces acteurs, les décideurs publics recourent aux $3 \mathrm{P}$ (prudence, prévention et précaution) dont la précaution semble à l'heure actuelle la dimension la plus mobilisée. En effet, la précaution n'implique pas de certitudes scientifiques au sens économique du terme (ce que suppose la prévention, comme nous l'avons montré) et ne s'inscrit pas dans un scénario pessimiste (ce qu'implique la prudence, comme nous l'avons décrit).

La précaution s'impose comme une logique dominante car, tenant compte des impératifs de la gouvernance, elle concilie les points de vue d'une pluralité d'acteurs en contexte d'incertitude et de controverse sociale. À ce titre, la précaution constitue une norme d'action publique. Elle requiert de prendre de la distance à l'égard des certitudes scientifiques et implique " d'agir en renonçant à une chimère, celle de vouloir disposer d'une information complète » (Van Griethuysen 2004 : 66).

Les pouvoirs publics sont confrontés à des situations complexes dans lesquelles il leur est difficile de placer le curseur entre la protection de la population et l'excès de précaution. En termes économiques, un excès de précaution pourrait nuire au développement de la technologie et à sa plus-value. Les pouvoirs publics doivent donc réguler le secteur de manière anticipée et proportionnée. En termes politiques, la stratégie des Régions qui cherchent à s'affirmer par rapport à l'Etat fédéral et à servir de caisse de résonance à des inquiétudes d'associations et de citoyens montre que la régulation publique ne se limite pas à la définition d'univers risqués ou incertains. Nous pouvons parler de gouvernance des risques dans le secteur de la téléphonie mobile en Belgique dans la mesure où différents acteurs sont associés au processus décisionnel en vue d'une approche la plus intégrée possible des risques.

Au sein de l'UCL, Caroline Deblander (caroline.deblander@uclouvain.be) est chercheur en sciences politiques, Nathalie Schiffino (nathalie.schiffino@uclouvain ) est professeur en sciences politiques et Louis Eeckhoudt est professeur ordinaire émérite en sciences économiques.

\section{BIBLIOGRAPHIE}

AFSSET. 2009. "Gouverner l'incertitude : les apports des sciences sociales à la gouvernance des risques sanitaires environnementaux, colloque organisé par l'Afsset et le Réseau Risques et Société », Paris. 
AFSSET. 2009a. «Avis de l'agence française de Sécurité Sanitaire de l'environnement et du travail concernant la mise à jour de l'expertise relative aux radiofréquences », http://www.leparticulier.fr/upload/docs/application/pdf/2009-

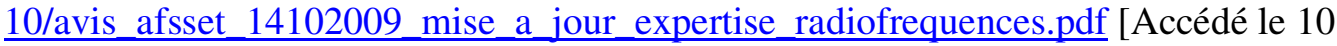
novembre 2009].

Beck, U. 1986. La société du risque. Sur la voie d'une autre modernité. Paris : Flammarion.

Bherer, L., J.-P. Collin, E. Kerrouche et Y. Pallard. 2005. Jeux d'échelles et transformation de l'Etat. Le gouvernement des territoires en France et au Québec. Québec : Les Presses de l'Université Laval.

Borraz, O. 2008. Les politiques du risque. Paris : Presses de la Fondation Nationale de Sciences Politiques.

Brenot, J, S. Bonnefous et P. Hubert. 1996. «Perception des risques nucléaires » Radioprotection, 31(4) : 515-528.

Burgess, A. 2004. Cellular phones, public fears, and a culture of precaution. Cambridge : Cambridge University Press.

Commission européenne. 2000. «Communication de la commission sur le recours au principe de précaution », COM (2000)1.

Debia, M. et J. Zayed. 2003. «Les enjeux relatifs à la perception et à la communication dans le cadre de la gestion des risques sur la santé publique », VertigO 4(1) : 1-8.

Dolowitz, D. et D. Marsh. 2000. «Learning from Abroad: The Role of Policy Transfer in Contemporary Policy-Making », Governance, 13(1) : 5-23.

Douglas, M. et A. Widalsky. 1982. Risk and culture. An essay on the selection of technological and environmental dangers. Los Angeles : University of California Press.

Dupont, Y. 2007. Dictionnaire des risques. Paris : Armand Colin.

EEA. 2008. Late lessons from early warnings: the precautionary principle 1896-2000. Copenhagen : European Environment Agency.

Ewald, F. 2009. «La mécanique diabolique du principe de précaution » Les Echos, 19.

Ewald, F. 1986. L' État-providence. Paris : Grasset.

Gilbert, C. 2003. «La fabrique des risques », Cahiers internationaux de sociologie, 1(114) : 55-72.

Godard, O. 1997. Le principe de précaution dans la conduite des affaires humaines. Paris : Editions de la Maison des Sciences de l'Homme.

Godard, O. 2000. «Le principe de précaution, une nouvelle logique de l'action entre science et démocratie », Philosophie Politique (11) : 17-56.

Godard, O. 2003. « Le principe de précaution comme norme de l'action publique, ou la proportionnalité en question », Revue Economique, 54(6) : 1245-1276.

Godard, O. 2007. «Le principe de précaution et la controverse OGM : les fondements éthiques de la décision collective » Revue de l'Institut d'Economie Publique, 21(2) : 13-75.

Fischhoff, B., S. Watson et C. Hope. 1984. «Defining Risk», Policy Sciences, 17 : 123-139.

Heywood, A. 2007. Politics. Houndmills : Palgrave MacMillan. 
Held, D. 2007. « De l'urgente nécessité de réformer la gouvernance globale », Recherches sociologiques et anthropologiques, 38(1) : 65-88.

Hergon, E. et al. 2004. «Les facteurs de perception et d'acceptabilité du risque : un apport pour la connaissance des représentations du risque transfusionnel » Transfusion Clinique et Biologique, 11: 130-137.

ICNIRP. 1998. « Guidelines for limiting exposure to time-varying electric, magnetic, and electromagnetic fields (up to $300 \mathrm{GHz}$ ) Health Physics, 74(4) : 494-522.

ICNIRP. 2009. «Guidelines on limits of exposure to static magnetic fields » Health Physics, 96(4) : 504-514.

Inter-Environnement Wallonie. 2008. «Synthèse des contributions. Consultation du Ministre du développement territorial sur l'implantation des antennes de téléphonie mobile en Région wallonne »http://www.iewonline.be/IMG/pdf/SYNTHESE_des_contributions.pdf [Accédé le 30 octobre 2008].

Jacob, S., et N. Schiffino. 2007. « Docteur Folamour apprivoisé? Les politiques publiques du risque »Politique et Sociétés, 26(2-3) : 45-72.

Jouve, B. 2007. «Le 'Political rescaling' pour théoriser l' État et la compétition territoriale en Europe », dans A. Faure et al. Action publique et changements d'échelles : les nouvelles focales du politique. Paris : L'Harmattan, p.45-56.

Kourilsky, P. 2002. Du bon usage du principe de précaution. Paris : Odile Jacob.

Latour, B. 2000. « Prenons garde au principe de précaution », Le Monde, 3 janvier.

November, A. et V. November. 2004. «Risque, assurance et irréversibilité » Revue Européenne de Sciences Sociales, 42 : 161-179.

Paillé, P., et A. Muchielli. 2010. L'analyse qualitative en sciences humaines et sociales. Paris : Armand Colin.

Parlement européen. 2008. «Projet de rapport sur les préoccupations quant aux effets pour la santé des champs électromagnétiques » (2008/2211(INI)).

Parlement européen. 2009. «Rapport sur les préoccupations quant aux effets pour la santé des champs électromagnétiques » A6-0089/2009.

Peretti-Watel, P. et J.-P. Moatti. 2009. Le principe de prévention. Paris : Editions du Seuil et La République des Idées.

Peretti-Watel, P. 2001. La société du risque. Paris : Editions La Découverte \& Syros. Rosenau, J.N., et E.-O. Czempiel (dirs.). 1992. Governance Without Government: Order and Change in World Politics. New York : Cambridge University Press.

SCENIHR. 2007. « Opinion on the possible effects of electromagnetic fields (EMF) on human health ».

Short, J.F. JR. 1984. « The social fabric at risk: toward the social transformation of risk analysis », American Sociological Association, 49 : 711-725.

Slovic, P. 1987. «Perception of risk », Science, $236: 280-285$.

Starr, C. 1969. « Social benefit versus technological risk. What is your safety willing to pay for safety », Science, 165 (19) : 1232-1238.

Van Griethuysen, P. 2004. "Pour une approche évolutive de la précaution », Revue Européenne de Sciences Sociales, 42 (130) : 35-70. 